OPEN ACCESS

Check for updates

\title{
Burden, treatment use, and outcome of secondary mitral regurgitation across the spectrum of heart failure: observational cohort study
}

\author{
Philipp E Bartko, ${ }^{1}$ Gregor Heitzinger, ${ }^{1}$ Noemi Pavo, ${ }^{1}$ Maria Heitzinger, ${ }^{2}$ Georg Spinka, ${ }^{1}$ \\ Suriya Prausmüller, ${ }^{1}$ Henrike Arfsten, ${ }^{1}$ Martin Andreas, ${ }^{3}$ Cornelia Gabler, ${ }^{4}$ Guido Strunk, ${ }^{5}$ \\ Julia Mascherbauer, ${ }^{1,6}$ Christian Hengstenberg, ${ }^{1}$ Martin Hülsmann, ${ }^{1}$ Georg Goliasch ${ }^{1}$
}

${ }^{1}$ Department of Internal

Medicine II, Medical University of

Vienna, Vienna, Austria

${ }^{2}$ Vienna University of Technology, Vienna, Austria

${ }^{3}$ Department of Cardiac Surgery, Medical University of Vienna,

Vienna, Austria

${ }^{4}$ IT Systems and

Communications, Medical

University of Vienna, Vienna,

Austria

${ }^{5}$ Complexity Research, Vienna, Austria

${ }^{6}$ Department of Internal Medicine 3, University Hospital St Pölten, Karl Landsteiner University of Health Sciences, St Pölten, Austria

Correspondence to: G Goliasch georg.goliasch@meduniwien.ac.a (or @ggoliasch on Twitter ORCID 0000-0002-6219-6104)

Cite this as: $B M J$ 2021;373:n1421 http://dx.doi.org/10.1136/bmj.n1421

Accepted: 2 June 2021

\section{ABSTRACT}

OBJECTIVES

To define prevalence, long term outcome, and treatment standards of secondary mitral regurgitation (sMR) across the heart failure spectrum.

DESIGN

Large scale cohort study.

SETTING

Observational cohort study with data from the Viennese community healthcare provider network between 2010 and 2020, Austria.

\section{PARTICIPANTS}

13223 patients with sMR across all heart failure subtypes.

\section{MAIN OUTCOME MEASURES}

Association between SMR and mortality in patients assigned by guideline diagnostic criteria to one of three heart failure subtypes: reduced, mid-range, and preserved ejection fraction, was assessed.

\section{RESULTS}

Severe SMR was diagnosed in 1317 patients (10\%), correlated with increasing age ( $\mathrm{P}<0.001)$, occurred across the entire spectrum of heart failure, and was most common in 656 (25\%) of 2619 patients with reduced ejection fraction. Mortality of patients with severe SMR was higher than expected for people of the same age and sex in the same community (hazard ratio $7.53 ; 95 \%$ confidence interval 6.83 to 8.30 , $\mathrm{P}<0.001)$. In comparison with patients with heart failure and no/mild sMR, mortality increased stepwise with a hazard ratio of 1.29 (95\% confidence interval 1.20 to $1.38, \mathrm{P}<0.001)$ for moderate and 1.82 (1.64

\section{WHAT IS ALREADY KNOWN ON THIS TOPIC \\ Secondary mitral regurgitation (SMR) is common in patients with heart failure and reduced ejection fraction and increases mortality \\ Knowledge about the prevalence of SMR and mortality across the whole heart failure spectrum is insufficient \\ Contemporary data on how available treatments for SMR are used in a state-of- the-art healthcare system are limited}

\section{WHAT THIS STUDY ADDS}

Secondary mitral regurgitation is highly prevalent among patients with heart failure and increasing with more impaired systolic function and ageing; severe mitral regurgitation is associated with excessive mortality and most pronounced in mid-range ejection fraction heart failure

Valve repair strategies are rarely used across the entire heart failure spectrum with SMR, despite availability of minimal invasive and low risk options to 2.02, P<0.001) for severe SMR. The association between severe SMR and excess mortality was consistent after multivariate adjustment and across all heart failure subgroups (mid-range ejection fraction: hazard ratio 2.53 (95\% confidence interval 2.00 to 3.19, P<0.001), reduced ejection fraction: 1.70 (1.43 to $2.03, \mathrm{P}<0.001)$, and preserved ejection fraction: 1.52 (1.25 to $1.85, \mathrm{P}<0.001)$ ). Despite available stateof-the-art healthcare, high volume heart failure, and valve disease programmes, severe sMR was rarely treated by surgical valve repair (7\%) or replacement (5\%); low risk transcatheter repair (4\%) was similarly seldom used.

CONCLUSION

Secondary mitral regurgitation is common overall, increasing with age and associated with excess mortality. The association with adverse outcome is significant across the entire heart failure spectrum but most pronounced in those with mid-range and reduced ejection fractions. Despite these poor outcomes, surgical valve repair or replacement are rarely performed; similarly, low risk transcatheter repair, specifically in the heart failure subsets with the highest expected benefit from treatment, is seldom used. The current data suggest an increasing demand for treatment, particularly in view of an expected increase in heart failure in an ageing population.

\section{Introduction}

Mitral regurgitation secondary to heart failure impairs quality of life, increases rates of hospital admission, and has a poor prognosis. ${ }^{1-3}$ These effects on individual patients have considerable challenges for care givers and service payers. Distributions and patterns of heart failure in the population have been well examined. ${ }^{45}$ Studies of mitral regurgitation, however, have focused on primary regurgitation, ${ }^{6-9}$ which is less prevalent and has different disease specific and epidemiological characteristics, making interpretation of outcomes and treatment standards for secondary mitral regurgitation (sMR) impossible. ${ }^{10} 11$ An important change in the understanding of heart failure and subsequently, sMR, has led to expanded definitions of the disease. ${ }^{12}$ In addition to reduced ejection fraction, these definitions now also cover the mid-range and preserved ejection fraction spectrum to capture the full extent of disease limiting generalisability of results from small selected historical cohorts. ${ }^{23}$ The clinical characteristics of patients with heart failure (advanced age, comorbidities, and intrinsic associated risk of 
heart failure) result in excessive procedural risks as a result of surgical management strategies, such as valve repair, or replacement; little use of traditional surgical techniques is therefore expected. Transcatheter mitral valve repair meets the clinical requirement for a low risk durable repair for severe sMR, but whether it is adequately used to reduce valvular driven heart failure remains unknown.

In this study we sought to describe the demographic characteristics of SMR-overall and according to subgroups of heart failure-to identify growing public healthcare demands. Furthermore, we aimed to describe the association between SMR and mortality according to subtypes of heart failure to identify populations that might be expected to improve with treatment. Moreover, this study sought to monitor current treatment of SMR in a population with stateof-the-art healthcare facilities, a full population health plan, and all available treatment options, from standard surgical valve repair and replacement to routinely used transcatheter mitral valve repair.

\section{Methods}

Study design, clinical measures, and follow-up

In this observational cohort study, we used the Medical University of Vienna longitudinal medical health records and echo database to identify all individuals with heart failure according to guideline definitions without relevant primary valve disease. This database covers all inpatients and outpatients from the Medical University of Vienna, core facilities of the Vienna healthcare alliance group with a public healthcare utility mandate for 1.5-1.9 million community residents during the study period. The definitions of heart failure cover all three types of heart failure: heart failure with reduced ejection fraction, with midrange ejection fraction, and with preserved ejection fraction. This methodology enabled full compliance with the guideline definitions of heart failure, which are different for the three ejection fraction ranges and include mandatory features for a correct diagnosis, such as relevant structural heart disease, diastolic dysfunction, signs and symptoms, and raised levels of natriuretic peptides. Detailed definitions of heart failure with reduced, mid-range, and preserved ejection fractions are specified in the guidelines. ${ }^{12}$ A detailed flow diagram of this study is shown in supplementary figure 1.

In patients with a left ventricular ejection fraction $\geq 40 \%$ at least one of the following criteria was required: relevant structural heart disease defined as left ventricular hypertrophy or left atrial enlargement, or diastolic dysfunction. In addition, a raised $\mathrm{N}$-terminal pro-brain natriuretic peptide (NT-proBNP) >125 $\mathrm{ng} / \mathrm{L}$ and signs and symptoms of heart failure were mandatory for inclusion in the study. In accordance with the guideline definitions of heart failure, no structural inclusion penalty was used in patients with a left ventricular ejection fraction $<40 \%$. Patients with evidence of significant primary valve disease were excluded from the analysis. In detail, these exclusions applied to the following criteria ${ }^{1}$ : evidence of primary/ organic mitral valve disease regardless of the degree of mitral regurgitation (prolapse, flail, perforation, or cleft of at least one leaflet; myxomatous and fibroelastic deficient leaflet remodelling, rheumatic valve disease, and endocarditis or congenital mitral valve disease; any mitral annular calcification with signs of mitral valve inflow obstruction or more than moderatesevere mitral annular calcification without stenotic effects) ${ }^{2}$; any other significant primary valve disease (greater than or equal to moderate) aortic stenosis, aortic regurgitation, pulmonary valve stenosis, or regurgitation; evidence of endocarditis/carcinoid valve disease; primary disease of the tricuspid valve).

The above described algorithm excluded 26986 patients owing to the absence of relevant structural heart disease and 4822 patients owing to primary valve disease. The remaining patient identification data were matched with the electronic health records to assess symptomatic and neurohumoral status of the patients to establish a definite diagnosis of heart failure. Patients without signs or symptoms of heart failure or NT-proBNP levels <125 ng/L $(n=6843)$ were subsequently excluded. Technically limited examinations with obscured degree of SMR or left ventricular ejection fraction, and focused examinations without sMR/left ventricular ejection fraction grading were also excluded $(n=1121)$. The final cohort included 13223 patients and was grouped according to subtypes of heart failure-that is, preserved left ventricular ejection fraction $\geq 50 \%$, mid-range left ventricular ejection fraction $40-49 \%$, and reduced left ventricular ejection fraction $<40 \%$. In addition, details of clinical variables, comorbidities, and clinical laboratory measurements were extracted from the electronic health records database using seed and targeted keyword search and designated coding from the international statistical classification of diseases and related health problems. Custom software developed by the Medical University of Vienna, the research documentation and data tool, was used for data extraction. Routine laboratory parameters were analysed from venous blood samples according to the local laboratory's standard procedure.

All cause mortality was defined as the primary endpoint. Mortality was determined from the Austrian Death Registry. Austrian law stipulates that deaths of all Austrian citizens (including those in foreign countries, if reported to Austrian officials) must be recorded in the central Austrian Death Registry, allowing almost complete follow-up of all patients. ${ }^{12}$ For each individual patient in the final study population, the corresponding average annual mortality rates by age and sex of the Austrian general population were obtained. These data were taken from the Austrian life tables of 2018, provided by the Austrian Statistical Office (Statistics Austria: www.statistik.at/web_en/ statistics/PeopleSociety/population). On the basis of these mortality data, the probability of cumulative expected survival was determined, which resulted in an expected survival curve as previously described. ${ }^{13}$ 
The study was approved by the institutional ethics review board.

\section{Echocardiographic examination}

All patients underwent a comprehensive echocardiographic examination according to the current guidelines. ${ }^{14}$ Transthoracic echocardiograms were obtained using commercially available equipment (Vivid E7 and E9, GE Healthcare, Chicago, IL and Acuson S2000, Siemens, Berlin, Germany) and interpreted by board certified physicians. Cardiac morphology was assessed using diameters in standard four and two chamber views. Left ventricular ejection fraction was calculated using the biplane Simpson method, and semiquantitative assessment of right heart function was performed by experienced readers using multiple acoustic windows and graded as normal, mild, moderate, and severe. Secondary mitral regurgitation was graded by an integrated approach comprising mitral valve morphology, width of the proximal regurgitant jet, proximal flow convergence, and pulmonary venous flow pattern, as previously described. ${ }^{2}$ Systolic pulmonary artery pressures were calculated by adding the peak tricuspid regurgitation systolic gradient to the estimated central venous pressure. Echocardiographic characteristics were extracted without alteration from the echocardiographic database.

\section{Statistical analysis}

Continuous data were presented as median and interquartile range. Cox proportional hazards regression analysis was applied to assess the effect of sMR (no/mild, moderate, severe) on survival and presented as hazard ratios with 95\% confidence intervals. Firstly, we conducted an unadjusted analysis with the severity of sMR as a single exploratory variable. We adjusted for a traditional clinical risk factor model (including age, sex, ischaemic heart disease, serum creatinine, mitral valve intervention, left ventricular end diastolic diameter, left ventricular function, and right ventricular function) and a bootstrap adjusted model. We used a stepwise bootstrap resampling procedure including all the variables presented in table 1 to identify the best fitting variables for this bootstrap model. Five hundred repeats with a $\mathrm{P}$ value of 0.05 for selection were performed, and variables selected in more than $95 \%$ of all repeats were included in the final bootstrap model (BMI, diabetes, chronic obstructive pulmonary disease, peripheral vascular disease, ischaemic heart disease, left ventricular function, blood urea nitrogen, bilirubin, albumin, and $\gamma$-glutamyltransferase). Correlations between the severity of sMR and any variables in the multivariate models were checked and judged as statistically non-significant. We tested for collinearity in the multivariable model using a variance inflation factor of less than 10 as the criterion for judging collinearity. The proportional hazards assumption was tested and satisfied in all instances using Schoenfeld residuals.
Only severe SMR is a potential target for further therapeutic intervention. Thus we focused the interaction analysis to assess the association of severe sMR (compared with patients with non-severe regurgitation) with outcome in heart failure and across the different types of heart failure (preserved, midrange, and reduced ejection fraction) in traditional established subgroups by entering interaction terms in the Cox proportional hazards regression models. The results of this subgroup analysis are presented as crude hazard ratios with 95\% confidence intervals.

Kaplan-Meier analysis (log rank test) was applied to assess differences in survival according to the severity of SMR. A two tailed P value less than 0.05 was regarded as statistically significant. SPSS 26.0, Stata 13.0, and R-3.6.2 were used for all statistical analyses.

\section{Patient and public involvement}

Neither study participants nor the public were involved in the study design, conduct, data analysis, reporting, or planning of dissemination of our research. We did not have contact with patients or members of the public with the level of statistical or methodological expertise to analyse or interpret our results.

\section{Results}

\section{Prevalence of sMR in heart failure}

Between 2010 and 2020, 52995 residents were included in the longitudinal echo database. Of these, 13223 residents met the eligibility criteria for heart failure according to the guideline definitions ${ }^{12} 1516$ and had no evidence of organic primary mitral valve disease or significant primary disease of another valve (supplementary fig 1). Among the total study population, sMR was absent/mild in 4007 (30\%), moderate in 7899 (60\%), and severe in 1317 (10\%) patients. The prevalence of moderate secondary mitral regurgitation rose with advancing age (fig 1 , top graph). The median age of the study population was 70 years (interquartile range 62-77), 8736 (66\%) patients were male, and the study participants had frequent but expected associated comorbidities (eg, hypertension in 8077 (61\%) patients, coronary artery disease in 6580 (50\%), and diabetes in 3407 (26\%)). Detailed baseline characteristics of the entire study population are displayed in table 1 .

With increasing severity of sMR, increases were seen in left ventricular end diastolic diameters (no/mild sMR $46 \mathrm{~mm}$ (interquartile range 42-51), moderate 47 $\mathrm{mm}$ (43-51), severe $53 \mathrm{~mm}$ (47-60)), the left atrial diameter (no/mild sMR $56 \mathrm{~mm}$ (53-60), moderate $59 \mathrm{~mm}$ (55-65), severe $65 \mathrm{~mm}$ (60-71)), and levels of NT-proBNP (mild/no sMR 633 ng/L (270-1843), moderate $1400 \mathrm{ng} / \mathrm{L}$ (531-3598), severe $3700 \mathrm{ng} / \mathrm{L}$ (1703-8224)). Severe sMR was more common in women $(n=525,12 \%)$ than in men $(n=792,9 \%)$. Of patients with moderate to severe sMR, 135 (1.5\%) of 9216 underwent mitral valve repair, 101 (1.1\%) mitral valve replacement, and $78(0.8 \%)$ transcatheter mitral valve repair over the total observation period. Detailed 


\begin{tabular}{|c|c|c|c|c|}
\hline Variables & All patients $(n=13223)$ & No/mild sMR $(n=4007)$ & $\begin{array}{l}\text { Moderate sMR } \\
(n=7899)\end{array}$ & Severe $s M R(n=1317)$ \\
\hline \multicolumn{5}{|l|}{ Type of heart failure: } \\
\hline HFpEF & $7362(56)$ & $2540(63)$ & $4491(57)$ & $331(25)$ \\
\hline HFmrEF & $3242(25)$ & $643(16)$ & $2269(29)$ & $330(25)$ \\
\hline HFrEF & $2619(20)$ & $824(21)$ & 1139 (14) & $656(50)$ \\
\hline \multicolumn{5}{|l|}{ Characteristics: } \\
\hline Median (interquartile range) age (years) & $70(62-77)$ & $67(58-75)$ & $72(63-78)$ & $71(61-78)$ \\
\hline Male & $8736(66)$ & $2882(72)$ & $5062(64)$ & $792(60)$ \\
\hline Median (interquartile range) body mass index & $27(25-31)$ & $28(25-32)$ & $27(24-31)$ & $26(24-29)$ \\
\hline \multicolumn{5}{|l|}{ Comorbidities: } \\
\hline Hypertension & $8077(61)$ & $2489(62)$ & $4911(62)$ & $677(51)$ \\
\hline Hyperlipidaemia & $4470(34)$ & $1444(36)$ & $2660(34)$ & $366(28)$ \\
\hline Diabetes & $3407(26)$ & $1106(28)$ & $2025(26)$ & $276(21)$ \\
\hline Coronary artery disease & $6580(50)$ & $2012(50)$ & $3927(50)$ & $641(49)$ \\
\hline Atrial fibrillation & $4012(30)$ & $803(20)$ & $2717(34)$ & $492(37)$ \\
\hline COPD & $1754(13)$ & $555(14)$ & $1024(13)$ & $175(13)$ \\
\hline Cerebral vascular disease & $2373(18)$ & $673(17)$ & 1477 (19) & $223(17)$ \\
\hline Peripheral vascular disease & $3167(24)$ & $958(24)$ & $1909(24)$ & $300(23)$ \\
\hline \multicolumn{5}{|l|}{ Echocardiographic characteristics: } \\
\hline Left ventricular end diastolic diameter & $47(43-52)$ & $46(42-51)$ & $47(43-51)$ & $53(47-60)$ \\
\hline Left atrial diameter $(\mathrm{mm})$ & $58(54-64)$ & $56(53-60)$ & $59(55-65)$ & $65(60-71)$ \\
\hline Right ventricular diameter $(\mathrm{mm})$ & $34(30-37)$ & $33(30-36)$ & $34(30-37)$ & $36(32-40)$ \\
\hline Right atrial diameter $(\mathrm{mm})$ & $57(52-63)$ & $54(51-59)$ & $57(53-63)$ & $61(56-68)$ \\
\hline Reduced right ventricular function (>moderate) & $350(2.7)$ & $78(2)$ & $154(2)$ & $118(9)$ \\
\hline Tricuspid regurgitation (>moderate) & $1465(11)$ & $135(3)$ & 839 (11) & $491(37.3)$ \\
\hline Median (interquartile range) pulmonary artery pressure $(\mathrm{mm} \mathrm{Hg})$ & $37(30-49)$ & $30(30-39)$ & $39.2(30.0-51.0)$ & $51.0(43.6-62.8)$ \\
\hline \multicolumn{5}{|l|}{ Laboratory measurements (median (interquartile range)): } \\
\hline Haemoglobin $(\mathrm{g} / \mathrm{L})$ & $128(111-141)$ & $131(114-145)$ & $126(110-140)$ & $123(106-138)$ \\
\hline White blood cell count $\left(10^{9} / \mathrm{L}\right)$ & $7.49(6.06-9.36)$ & $7.67(6.16-9.61)$ & $7.39(6.00-9.23)$ & $7.65(6.16-9.41)$ \\
\hline C reactive protein $(\mathrm{mg} / \mathrm{L})$ & $7.70(2.50-26.30)$ & $7.80(2.60-27.70)$ & $7.50(2.30-25.20)$ & $8.90(2.90-28.60)$ \\
\hline Creatinine $(\mu \mathrm{mol} / \mathrm{L})$ & $92.82(76.91-118.46)$ & $89.29(74.26-112.27)$ & $93.71(77.80-120.23)$ & $98.13(80.45-129.07)$ \\
\hline Blood urea nitrogen $(\mathrm{mmol} / \mathrm{L})$ & $6.79(5-9.29)$ & $6.07(4.64-8.57)$ & $6.79(5-9.64)$ & $7.5(5.71-11.07)$ \\
\hline Albumin $(\mathrm{g} / \mathrm{L})$ & $39(34-42)$ & $39(35-43)$ & $39(34-42)$ & $37.9(33.5-41.6)$ \\
\hline Bilirubin $(\mu \mathrm{mol} / \mathrm{L})$ & $10.26(6.84-15.39)$ & $10.26(6.84-15.39)$ & $10.26(6.84-15.39)$ & $11.97(8.55-18.81)$ \\
\hline Aspartate transaminase $(\mathrm{U} / \mathrm{L})$ & $26(20-37)$ & $26(20-37)$ & $26(20-36)$ & $27(21-38)$ \\
\hline Alanine transaminase $(\mathrm{U} / \mathrm{L})$ & $24(17-37)$ & $25(18-38)$ & $24(17-36)$ & $24(17-41)$ \\
\hline Y-glutamyltransferase $(\mathrm{U} / \mathrm{L})$ & $40(23-81)$ & $36(22-74)$ & $39(23-78)$ & $58(33-115)$ \\
\hline Total cholesterol (mmol/L) & $4.11(3.31-4.99)$ & $4.19(3.41-5.10)$ & $4.11(3.31-4.97)$ & $3.91(3.10-4.73)$ \\
\hline NT-proBNP (ng/l) & $1223(436-3452)$ & $633(270-1843)$ & $1400(531-3598)$ & $3700(1703-8224)$ \\
\hline \multicolumn{5}{|l|}{ Mitral valve intervention within observation period: } \\
\hline Mitral valve repair & $138(1.04)$ & $3(0.07)$ & $44(0.56)$ & $91(6.91)$ \\
\hline Mitral valve replacement & $108(0.82)$ & $7(0.17)$ & $39(0.49)$ & $62(4.71)$ \\
\hline Transcatheter mitral valve repair & $79(0.60)$ & $1(0.02)$ & $31(0.39)$ & $47(3.57)$ \\
\hline
\end{tabular}

$\mathrm{COPD}=$ chronic obstructive pulmonary disease; $\mathrm{HFmrEF=heart} \mathrm{failure} \mathrm{with} \mathrm{mid-range} \mathrm{ejection} \mathrm{fraction} \mathrm{(40-49 \% );} \mathrm{HFpEF=heart} \mathrm{failure} \mathrm{with} \mathrm{preserved} \mathrm{ejection} \mathrm{fraction} \mathrm{(} 250 \%)$; HFrEF=heart failure with reduced ejection fraction ( $440 \%)$; NT-proBNP=N-terminal pro-brain natriuretic peptide; SMR=secondary mitral regurgitation.

Tables showing the degree of secondary mitral regurgitation according to each heart failure subgroup are in the online supplementary appendix.

baseline characteristics according to severity of mitral regurgitation are presented in table 1.

Among the 13223 patients with heart failure, 7362 (56\%) patients presented with preserved, $3242(25 \%)$ with mid-range, and 2619 (20\%) with reduced ejection fractions. Severe sMR was most commonly encountered in patients with reduced ejection fraction, with an observed prevalence of $25 \%$ ( $n=656)$, compared with $10 \%(n=330)$ in patients with mid-range and $4.5 \%$ $(\mathrm{n}=331)$ in patients with preserved ejection fraction (fig 1, lower panel). Detailed baseline characteristics according to type of heart failure and severity of mitral regurgitation are presented in supplementary table $1 \mathrm{~A}$ C. Briefly, the ventricular component of severe sMR was more pronounced in patients with reduced ejection fraction as illustrated by a larger left ventricular end diastolic diameter (58 mm (interquartile range 52-64)) as compared with patients with mid-range ( $51 \mathrm{~mm}$ (4756)) and preserved (46 mm (42-50)) ejection fractions, as well as a higher prevalence of ischaemic heart disease (reduced 59\%, mid-range 62\%, preserved 41\% ejection fractions). Mitral valve interventions were less commonly performed in patients with heart failure and preserved ejection fraction $(2.2 \%, n=163)$ over the observation period, followed by mid-range $(2.5 \%$, $\mathrm{n}=80)$ and reduced $(3.1 \%, \mathrm{n}=82)$ ejection fractions.

\section{Outcome of SMR in heart failure}

During a median follow-up time of 60 months (interquartile range 30-87), 4330 (33\%) patients died. The proportion of observed fatal events at four years for patients with heart failure with severe SMR was $39 \%$, and $25 \%$ for those with no/mild sMR (compared with an expected $2 \%$ rate of fatal events in the age 

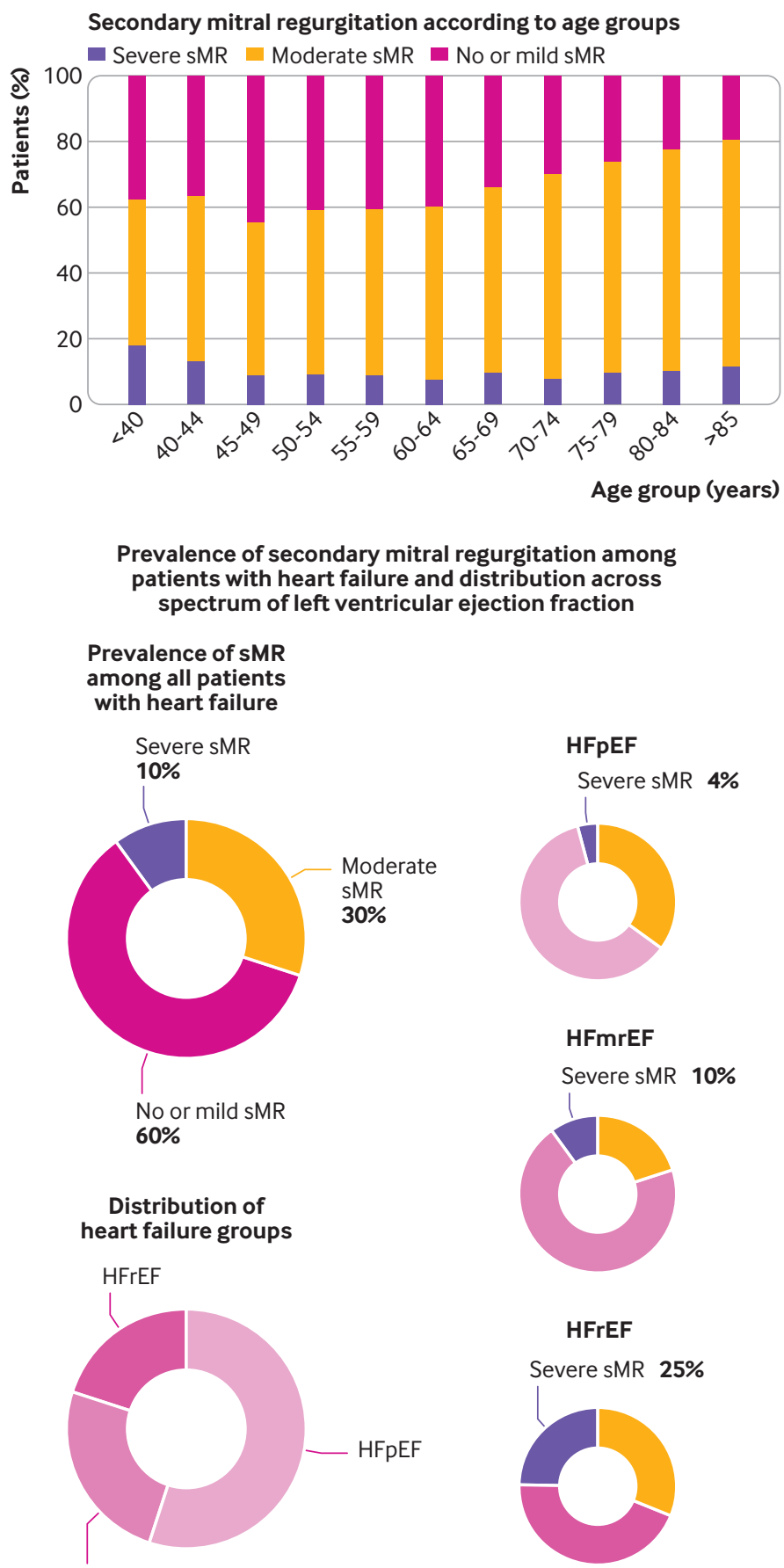

HFmrEF

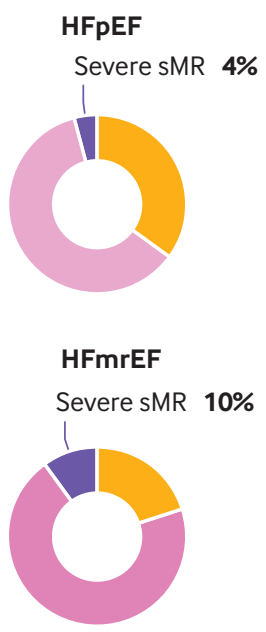

HFrEF
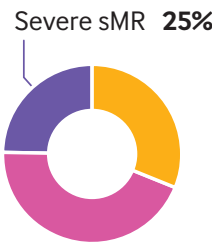

Fig 1 | Prevalence of secondary mitral regurgitation in patients with heart failure stratified by age. (Upper panel) Prevalence of no/mild, moderate, and severe secondary mitral regurgitation according to age groups in heart failure. (Lower panel) Prevalence of secondary mitral regurgitation overall and according to heart failure type (preserved, mid-range, and reduced ejection fractions). The prevalence of severe secondary mitral regurgitation is most substantial among patients with reduced ejection fraction followed by mid-range and preserved ejection fractions. Heart failure subtypes: $\mathrm{HFmrEF}=$ mid-range ejection fraction; $\mathrm{HFpEF}=$ preserved ejection fraction; $\mathrm{HFrEF}=$ reduced ejection fraction. $\mathrm{SMR}=$ secondary mitral regurgitation

and sex matched community) and at eight years for patients with heart failure with severe sMR was 55\%, and $37 \%$ for those with no/mild sMR ( $v$ an expected $14 \%$ rate of fatal events in the age and sex matched community, $\mathrm{P}<0.001$, fig 2). Compared with the

expected survival for people of the same age and sex, an excess mortality was seen in patients with moderate sMR (hazard ratio 5.08, 95\% confidence interval 4.78 to $5.40, \mathrm{P}<0.001)$ and patients with severe sMR $(7.53$, 6.83 to $8.30, \mathrm{P}<0.001)$. Compared with patients with heart failure with no/mild sMR as disease controls, a stepwise increase in risk for patients with heart failure dependent on the severity of SMR was seen with an unadjusted hazard ratio of 1.29 (95\% confidence interval 1.20 to $1.38, \mathrm{P}<0.001$ ) for moderate and 1.82 (1.64 to $2.02, \mathrm{P}<0.001$ ) for severe regurgitation. The results remained virtually unchanged after multivariable adjustment using a traditional clinical risk factor model and a bootstrap adjusted model (table 2).

The significant adverse impact of severe sMR in patients with heart failure was consistent across all the examined subgroups (fig 3), except for patients with severely reduced right ventricular function $(\mathrm{P}=0.7)$. We observed significant interactions between severe SMR and hypertension, ischaemic heart disease, and right ventricular function. The association between severe SMR and all cause mortality was more pronounced in patients with a history of hypertension (yes: hazard ratio $1.76,95 \%$ confidence interval 1.59 to 2.00, $\mathrm{P}<0.001$; no: 1.32, 1.13 to 1.54, $\mathrm{P}<0.001$; $\mathrm{P}$ for interaction $<0.01$ ), ischaemic heart disease (yes: 1.89 , 1.68 to $2.13, \mathrm{P}<0.001$; no: $1.20,1.05$ to $1.39, \mathrm{P}=0.01$; $\mathrm{P}$ for interaction $<0.001$ ), and attenuated in patients with severely impaired right ventricular function (yes: $0.94,0.68$ to $1.30, \mathrm{P}=0.70$; no: $1.53,1.39$ to 1.68 , $\mathrm{P}<0.001$; $\mathrm{P}$ for interaction 0.01 ).

The intrinsic risk of heart failure represented by patients with no/mild sMR was high overall and across all heart failure subsets (fig 4, violet red dashed lines, $\log$ rank $\mathrm{P}<0.001)$. Analogously to the overall analysis, we confirmed a stepwise increase of risk dependent on the severity of sMR across all types of heart failure (table 2) compared with people of the same age and sex in the same community and compared with patients with heart failure with no/mild sMR (fig 4).

The significant adverse effect of severe sMR in patients with heart failure and preserved, mid-range and reduced ejection fractions was consistent across all the examined subgroups. Excess mortality of severe SMR was most pronounced in patients with heart failure and mid-range ejection fraction (hazard ratio 2.53, 95\% confidence interval 2.00 to $3.19, \mathrm{P}<0.001$ ) and reduced ejection fraction $(1.70,1.43$ to 2.03 , $\mathrm{P}<0.001)$, followed by preserved ejection fraction (1.52, 1.25 to $1.85, \mathrm{P}<0.001)$ in comparison with patients with heart failure and no/mild sMR. Detailed subanalyses assessing the adverse effect of severe sMR in patients with heart failure and preserved, midrange, and reduced ejection fraction are displayed in supplementary figure $2 \mathrm{~A}-\mathrm{C}$.

\section{Discussion}

These data provide new information on the prevalence, outcome, and treatment of sMR across the entire spectrum of heart failure. The analyses 


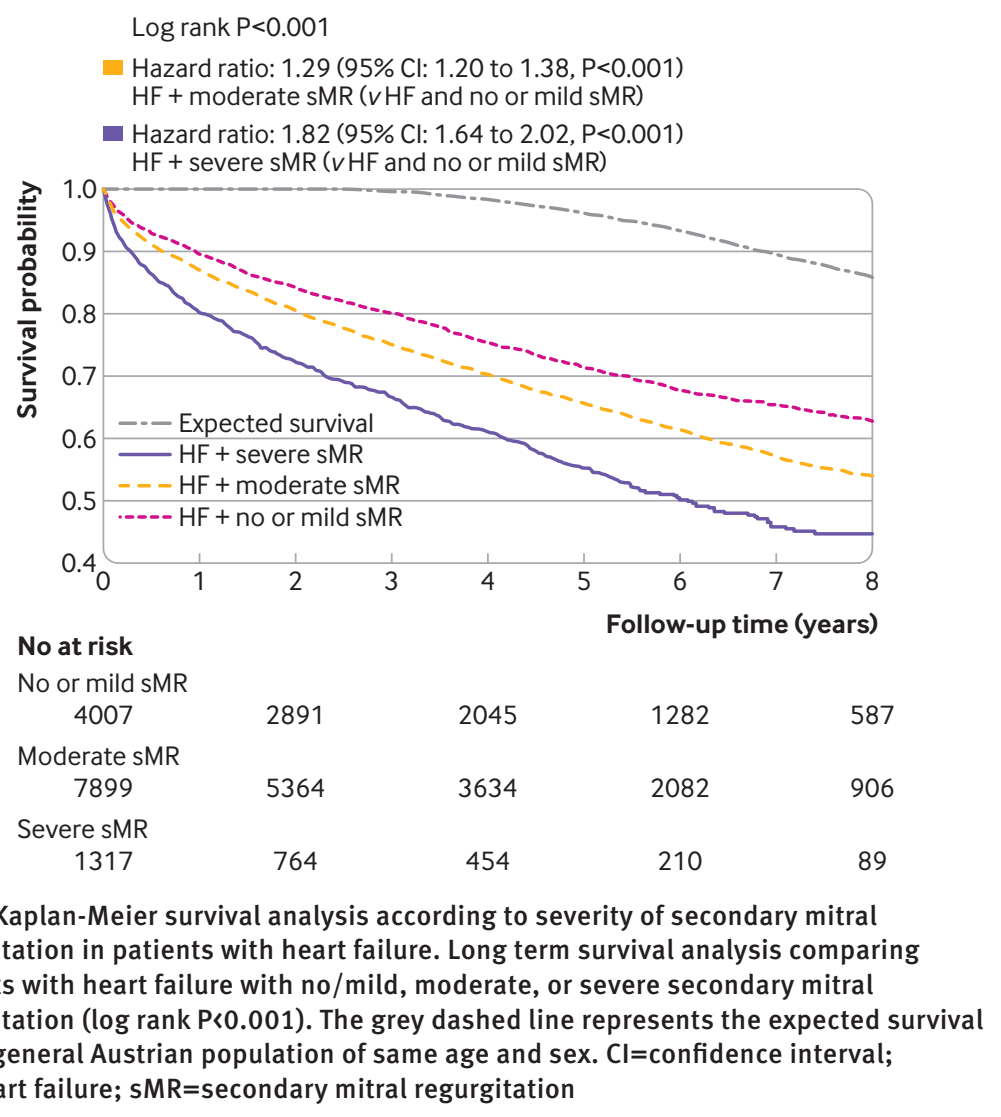

examine these aspects in a large representative cohort of patients with granular and comprehensive diagnostic criteria for heart failure, setting a standard for international comparison, monitoring of tailored treatment programmes, and planning of public health policy. Moderate and severe SMR are highly prevalent and expected to rise with the projected increase of heart failure in an ageing population. Severe sMR is associated with excess mortality, with a fatal event rate of $55 \%$ eight years after diagnosis. Nonetheless, surgical treatment of sMR is performed rarely despite available state-of-the-art healthcare facilities, a high volume of heart failure and valve disease programmes, a low barrier to health service access, and a complete population health plan coverage. Transcatheter mitral repair-which meets the clinical need for a low risk treatment in these vulnerable patients-is also performed infrequently across the entire spectrum of heart failure, and rarely used in those patients who might be expected to benefit most.

Given an overall heart failure prevalence of $1-2 \%$ in the community, ${ }^{17}$ in line with the present data that cover a significant proportion of $1 \%$ of the community, the proportion of individuals in the community affected is projected to be at least $0.08-0.16 \%$ overall for severe sMR and 0.6-1.2\% overall for moderate and severe SMR, indicating that 2.6-5.2 million Europeans have moderate or severe sMR. The increasing prevalence of heart failure in the elderly, and the high proportion of patients at risk of advancing from moderate to severe sMR, ${ }^{18}$ indicate an exponential public health threat. Even throughout the decade of the study, the population of those aged over 40 increased by roughly $1 \%$. These projections are worrisome and crucial for healthcare providers, service payers, and researchers, in order to account for the cost of public health and potential ways to deal with these imminent threats to the healthcare system.

Valve repair or replacement, is even less frequently deployed in SMR than in primary mitral regurgitation, previously reported as undertreated. ${ }^{6}$ These observations are remarkable in a community with readily available state-of-the-art healthcare facilities, including high volume heart failure and valve disease programmes, together with easy access to health services and a complete population health plan. Valve repair and replacements are rarely performed owing mainly to the excessive risks associated with

\begin{tabular}{|c|c|c|c|c|c|c|c|}
\hline \multirow[b]{2}{*}{ Observed sMR grade } & \multirow{2}{*}{$\begin{array}{l}\text { No of patients/ } \\
\text { events }\end{array}$} & \multicolumn{2}{|l|}{ Univariable model } & \multicolumn{2}{|l|}{ Bootstrap adjusted model* } & \multicolumn{2}{|l|}{ Clinical risk factor adjusted model $t$} \\
\hline & & Crude hazard ratio $(95 \% \mathrm{Cl})$ & P value & Adjusted hazard ratio $(95 \% \mathrm{Cl})$ & $P$ value & Adjusted hazard ratio $(95 \% \mathrm{Cl})$ & $P$ value \\
\hline Total study population: & $13223 / 4330$ & & & & & & \\
\hline No/mild sMR & $4007 / 1129$ & Reference & & Reference & & Reference & \\
\hline Moderate SMR & $7899 / 2668$ & $1.29(1.20$ to 1.38$)$ & $<0.001$ & $1.17(1.08$ to 1.26$)$ & $<0.001$ & $1.08(1.01$ to 1.16$)$ & 0.04 \\
\hline Severe sMR & $1317 / 533$ & 1.82 (1.64 to 2.02$)$ & $<0.001$ & $1.45(1.29-1.64)$ & $<0.001$ & 1.49 (1.32 to 1.67$)$ & $<0.001$ \\
\hline HFpEF: & $7362 / 2314$ & & & & & & \\
\hline No/mild sMR & $2540 / 752$ & Reference & & Reference & & Reference & \\
\hline Moderate sMR & $4491 / 1443$ & $1.15(1.06$ to 1.26$)$ & 0.001 & 1.07 (0.97 to 1.18$)$ & 0.18 & $1.00(0.92$ to 1.10$)$ & 0.94 \\
\hline Severe sMR & $331 / 119$ & $1.52(1.25$ to 1.85$)$ & $<0.001$ & $1.30(1.04$ to 1.61$)$ & 0.02 & $1.49(1.21$ to 1.84$)$ & $<0.001$ \\
\hline HFmrEF: & $3242 / 1036$ & & & & & & \\
\hline No/mild sMR & $643 / 139$ & Reference & & Reference & & Reference & \\
\hline Moderate sMR & $2269 / 755$ & 1.65 (1.38 to 1.98$)$ & $<0.001$ & $1.41(1.13$ to 1.75$)$ & 0.002 & 1.30 (1.08 to 1.57$)$ & 0.006 \\
\hline Severe sMR & $330 / 142$ & $2.53(2.00$ to 3.19$)$ & $<0.001$ & $2.00(1.51$ to 2.65$)$ & $<0.001$ & 1.94 (1.50 to 2.52$)$ & $<0.001$ \\
\hline HFrEF: & $2619 / 980$ & & & & & & \\
\hline No/mild sMR & $824 / 238$ & Reference & & Reference & & Reference & \\
\hline Moderate sMR & $1139 / 470$ & 1.61 (1.38 to 1.88$)$ & $<0.001$ & 1.41 (1.18 to 1.69$)$ & $<0.001$ & 1.38 (1.17 to 1.62$)$ & $<0.001$ \\
\hline Severe sMR & $656 / 272$ & $1.70(1.43$ to 2.03$)$ & $<0.001$ & 1.54 (1.26 to 1.88$)$ & $<0.001$ & $1.50(1.24$ to 1.81$)$ & $<0.001$ \\
\hline
\end{tabular}




\begin{tabular}{|c|c|c|c|}
\hline Subgroup & $\begin{array}{c}\text { Hazard ratio } \\
(95 \% \mathrm{Cl})\end{array}$ & $\begin{array}{c}\text { Hazard ratio } \\
(95 \% \mathrm{Cl})\end{array}$ & $\begin{array}{c}\mathrm{P} \text { for } \\
\text { interaction }\end{array}$ \\
\hline All patients & $-\diamond-$ & 1.53 (1.40 to 1.68$)$ & \\
\hline Age (years) & & & 0.77 \\
\hline$\geq 70$ & $\longrightarrow$ & 1.58 (1.41 to 1.77$)$ & \\
\hline$<70$ & $\longrightarrow-$ & $1.52(1.30$ to 1.77$)$ & \\
\hline Sex & & & 0.07 \\
\hline Female & $\longrightarrow-$ & 1.37 (1.17 to 1.59$)$ & \\
\hline Male & $-\bullet-$ & 1.64 (1.47 to 1.84 ) & \\
\hline Hypertension & & & 0.007 \\
\hline Yes & $\leftrightarrow-$ & 1.76 (1.59 to 2.00$)$ & \\
\hline No & $\longrightarrow-$ & $1.32(1.13$ to 1.54$)$ & \\
\hline Diabetes & & & 0.42 \\
\hline Yes & $\longrightarrow \diamond$ & 1.50 (1.27 to 1.78$)$ & \\
\hline No & $-\diamond-$ & 1.59 (1.43 to 1.77$)$ & \\
\hline Atrial fibrillation & & & 0.77 \\
\hline Yes & $\longrightarrow-$ & 1.55 (1.35 to 1.79$)$ & \\
\hline No & $-\diamond-$ & $1.47(1.30$ to 1.66$)$ & \\
\hline Ischaemic heart disease & & & $<0.001$ \\
\hline Yes & $-\diamond-$ & 1.89 (1.68 to 2.13$)$ & \\
\hline No & $\longrightarrow-$ & 1.20 (1.05 to 1.39$)$ & \\
\hline LV end diastolic diameter (mm) & & & 0.86 \\
\hline$\geq 47$ & $-\diamond-$ & $1.62(1.46$ to 1.81$)$ & \\
\hline$<47$ & $\longrightarrow-$ & 1.57 (1.31 to 1.90$)$ & \\
\hline LA diameter (mm) & & & 0.86 \\
\hline$\geq 58$ & $-\diamond-$ & 1.41 (1.27 to 1.56$)$ & \\
\hline$<58$ & $\longrightarrow \longrightarrow$ & 1.36 (1.07 to 1.73$)$ & \\
\hline RV end diastolic diameter (mm) & & & 0.33 \\
\hline$\geq 34$ & $-\diamond-$ & 1.54 (1.38 to 1.72$)$ & \\
\hline$<34$ & $\longrightarrow-$ & 1.40 (1.18 to 1.66$)$ & \\
\hline RV function & & & 0.012 \\
\hline$\leq$ moderately reduced RVF & $-\diamond-$ & 1.53 (1.39 to 1.68$)$ & \\
\hline Severely reduced RVF & $\diamond$ & 0.94 (0.68 to 1.30$)$ & \\
\hline Creatinine & & & 0.35 \\
\hline$\geq 1.05 \mathrm{mg} / \mathrm{dL}$ & $-\diamond-$ & 1.56 (1.39 to 1.74$)$ & \\
\hline$<1.05 \mathrm{mg} / \mathrm{dL}$ & $\longrightarrow-$ & 1.40 (1.18 to 1.65$)$ & \\
\hline & $1.5 \quad 2.0$ & 5 & \\
\hline
\end{tabular}

Fig 3 | Subgroup analysis of long term mortality for patients with heart failure and severe secondary mitral regurgitation. Univariable Cox regression analyses using the median values of the total study population as cut off points for continuous data. The $P$ value for interaction refers to an interaction between severe secondary mitral regurgitation and the respective subgroup. Secondary mitral regurgitation outcome according to heart failure type. $\mathrm{Cl}=$ confidence interval; $\mathrm{LA}=$ left atrium; $\mathrm{LV}=$ left ventricle; $\mathrm{RV}=$ right ventricle; $\mathrm{RVF}=$ right ventricular function

surgical treatment, particularly in elderly patients with heart failure, who have an expected high level of comorbidities. ${ }^{4}$ The standardised approach to surgical risk assessment in patients with valve disease during multidisciplinary heart team meetings is the use of risk calculation tools based on observational data, in which age, comorbidities, history of heart failure, and systolic dysfunction are substantial components of the score, indicating intrinsically prohibitive surgical risk in most patients with sMR. ${ }^{10}{ }^{11}$ In addition, the lack of proof that surgical valve repair or replacement can improve survival in $\mathrm{sMR}^{1920}$ justifies a conservative surgical approach restricted to those for whom another cardiac procedure, such as bypass grafting, is vital. ${ }^{21}$
In contrast to the surgical approach, treatments such as transcatheter mitral valve repair, ${ }^{10} 1122$ tailored to meet the demand of high risk populations, have been shown to improve symptoms, hospital admissions for heart failure, and outcome. ${ }^{22}$ These treatments were available throughout the study but rarely performed across the entire range of heart failure, particularly in patients with mid-range and preserved ejection fractions. The reasons for the sparse use of these therapeutic options in patients with heart failure and mid-range and preserved ejection fractions become evident upon closer investigation: the treatments have neither been tested in a controlled manner nor are there solid data on the effect of sMR in these subtypes 
Heart failure with preserved LVEF

Log rank $\mathrm{P}<0.001$

Hazard ratio: 1.52 (95\% Cl: 1.25 to $1.85, \mathrm{P}<0.001$ )

HFpEF + severe sMR ( $v$ HFpEF and no or mild sMR)

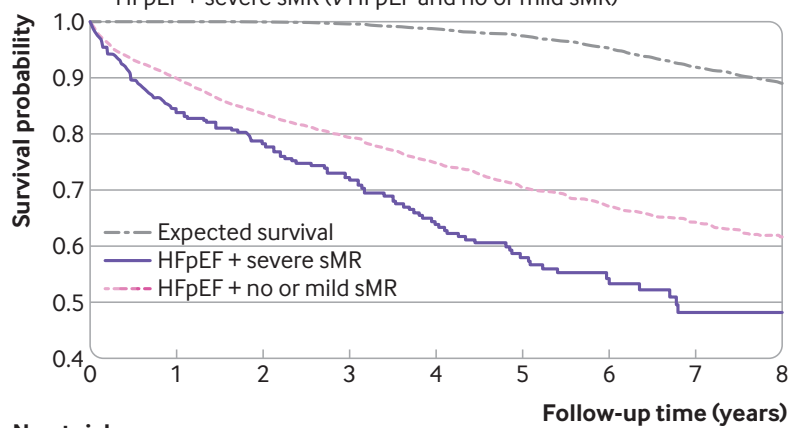

No at risk

No or mild sMR

2540

Severe sMR

331

1845

203

119

54

19

Heart failure with mid-range LVEF

Log rank $P<0.001$

- Hazard ratio: 2.53 (95\% Cl: 2.00 to 3.19, $\mathrm{P}<0.001$ )

$\mathrm{HFmrEF}+$ severe sMR ( $v$ HFmrEF and no or mild sMR)

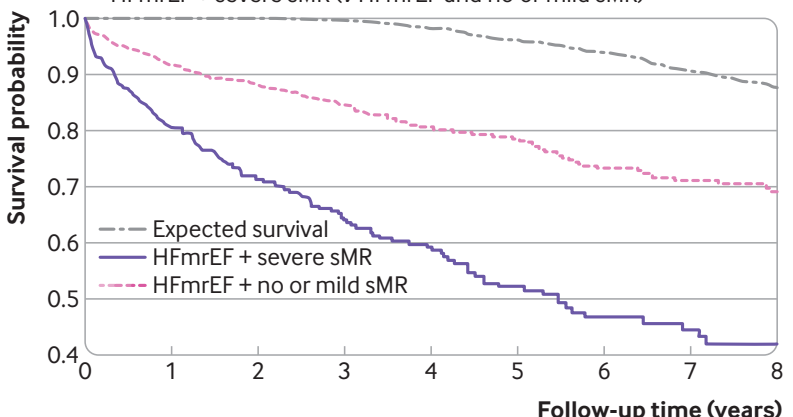

No at risk

No or mild sMR

$\begin{array}{lllll}643 & 472 & 318 & 186\end{array}$

Severe sMR

330

192

109

49

25

Heart failure with reduced LVEF

Log rank $P<0.001$

- Hazard ratio: 1.70 (95\% Cl: 1.43 to 2.03, $P<0.001$ )

HFrEF + severe sMR ( $v$ HFrEF and no or mild sMR)

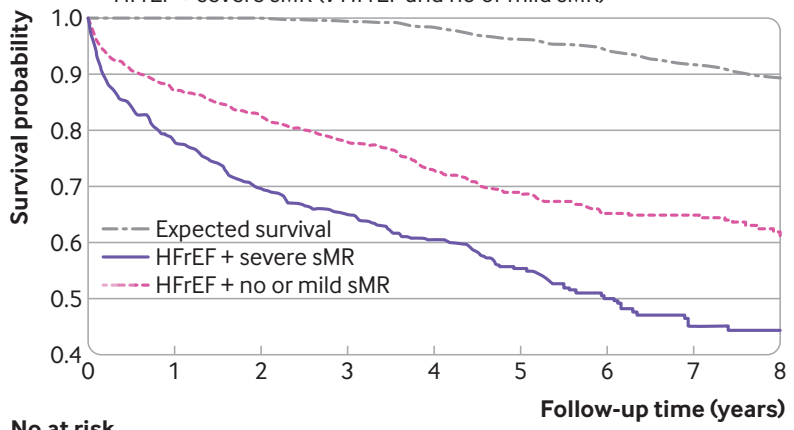

No at risk

No or mild sMR

$\begin{array}{ccccc}824 & 574 & 378 & 230 & 103 \\ \begin{array}{c}\text { vere sMR } \\ 656\end{array} & 372 & 226 & 106 & 45\end{array}$

Fig 4 | Kaplan-Meier survival analysis in patients with severe secondary mitral regurgitation across the spectrum of heart failure. Long term survival analysis of patients with heart failure and severe secondary mitral regurgitation compared with patients with heart failure and no/mild secondary mitral regurgitation as disease controls in those with preserved (log rank $P<0.001)$, mid-range (log rank $P<0.001)$, and reduced (log rank $P<0.001)$ ejection fractions. The grey dashed line represents the expected survival of the general Austrian population of same age and sex. Heart failure subtypes: $\mathrm{HFmrEF=mid-range} \mathrm{ejection} \mathrm{fraction;} \mathrm{HFpEF}=$ preserved ejection fraction; $\mathrm{HFrEF=reduced} \mathrm{ejection}$ fraction. $\mathrm{Cl}=$ confidence interval; $\mathrm{LVEF}=$ left ventricular ejection fraction; sMR=secondary mitral regurgitation 
of heart failure. ${ }^{23}$ As a consequence, current guideline recommendations for transcatheter mitral valve repair are more restricted than recommendations for primary mitral regurgitation. ${ }^{21} \mathrm{~A}$ third randomised trial, the RESHAPE-HF2 trial (ClinicalTrials.gov identifier: NCT02444338), currently enrolling, might clarify whether or not treatment benefit with an expanded ejection fraction spectrum up to $45 \%$ can be expected.

Before considering an intervention, initiation and optimisation of medical treatment is important in patients with heart failure. ${ }^{12}$ In patients with reduced ejection fraction, in particular, medical treatment might have a strong impact on mortality, as shown by several randomised trials. In our cohort, the association of sMR with survival is congruent with previous studies of patients receiving guideline directed medical treatment, ${ }^{2}$ supporting the applicability to current clinical practice. It should be noted, however, that adherence to medical treatment is generally low and fewer than $1 \%$ of patients with reduced ejection fraction are treated with target doses of $\beta$ blockers, renin-angiotensin-aldosterone system antagonists. In patients with mid-range and preserved ejection fractions, the effect of medical treatment is less clear and unlikely to affect the observed excess mortality rates in patients with severe sMR.

Our data provide insight into the outcome of sMR, comparing the association between sMR and mortality with the expected risk of the standard population, and examining background risk inherent to heart failure overall and according to the respective subtype. The key observation is that SMR is associated with excess mortality overall and across the entire range of heart failure. The association of sMR with mortality persisted even after adjustment for variables based on clinical experience (clinical risk factor model) and an independent variable selection procedure to adjust for the most comprehensive set of variables associated with adverse outcome among all recorded variables (bootstrap adjusted model). With a fatal event rate of $55 \%$ eight years after diagnosis, the prognosis associated with severe sMR is poor for all the underlying heart failure subtypes (fig 4, purple lines). Any of the heart failure subtypes, however, is tied to an intrinsic risk of mortality (fig 4, violet red lines). Thus the risk of SMR is largest in patients with mid-range ejection fraction followed by those with reduced and preserved ejection fractions. In other words, these data indicate that over the past decade, despite readily available low risk treatments, clinical compassionate use and randomised trials ${ }^{2224}$ have not targeted the patients with the highest expected benefit from treatment, but rather those for whom an expected benefit might be elusive owing to a competing risk (heart failure $v$ SMR). 2224

These observations continue to amplify a previous concept ${ }^{2}$ that refined the prognostic effect of sMR in patients with a reduced ejection fraction, showing that, in advanced stages of heart failure, the prognostic effect of SMR is limited, whereas in less advanced stages, sMR is an important driving factor of mortality-a possible explanation for the difference between two randomised clinical transcatheter mitral valve repair trials. $^{22}{ }^{24}$ Furthermore, subgroup analysis indicates an interaction between severe sMR and impaired right ventricular function, which might need consideration. Moreover, subgroup analysis shows an excessive risk for the concomitant presence of severe sMR and hypertension, identifying a vulnerable subgroup with a potentially modifiable risk factor. Otherwise, severe sMR was associated with excess mortality across all subgroups (fig 3). A previous study suggested a similar effect of sMR in patients with reduced ejection fraction regardless of the aetiology, ${ }^{3}$ partly contradicting the current guideline recommendation for mitral valve repair or replacement only if another cardiac surgical procedure is planned. The current data, however, show a more pronounced impact of ischaemic sMR, strengthening the evidence for the current indications to add surgical repair when coronary bypass graft surgery is planned. ${ }^{15} 16$

\section{Implications for patient management:}

Our data show the importance of echocardiographic screening and monitoring in patients with suspected heart failure. Echocardiography is not only mandatory for diagnosis of heart failure, including subclassification, but also a cost efficient, safe, and widely available diagnostic tool that identifies SMR and its associated risk of mortality, which is high if severe. Indeed, patients with severe sMR are a high risk subgroup in need of clinical attention. This usefulness of echocardiographic screening has previously been recognised exclusively in specific heart failure subtypes (reduced ejection fraction), but our data show this across the entire spectrum with similar detrimental associations in every heart failure subtype (fig 2 and fig 4). Figure 1 (lower panel) shows that the overall proportion of patients with severe sMR is $10 \%$. In daily clinical routine, patients with severe sMR and preserved and mid-range ejection fractions will be as common as those with reduced ejection fraction. In addition to screening and monitoring, these results suggest the need for early involvement of heart teams with integrated and complementary care.

\section{Strengths and limitations of this study}

The results of our study have several specific strengths: firstly, they provide a comprehensive diagnosis of heart failure and the specific subtypes according to societal guideline diagnostic criteria $^{12}$; secondly, the sample size is 25 times larger overall and seven times larger in those with severe sMR than in the most recent community cohort study of mitral regurgitation ${ }^{6}$; thirdly, the availability of transcatheter mitral valve repair during the study period in contrast to previous US cohort studies, ${ }^{689}$ in which transcatheter repair was not approved for sMR and with Food and Drug Administration approval in 2019, a 10 year community experience of heart failure as in our study might be expected in 2030 at the earliest; fourthly, full coverage of the entire spectrum of heart failure, including a large 
proportion of patients with moderate and no/mild SMR, indicating the background risk of heart failure without SMR, and the graded association between SMR and outcome.

A potential limitation of the study is that undiagnosed heart failure, and therefore sMR, is not considered. Given that signs and symptoms are a prerequisite of heart failure according to the guideline definition, the proportion of undiagnosed patients is probably low but subject to the random sampling of general population based studies. Our study was not designed to estimate the incidence and prevalence of heart failure, but given the large sample, the proportions of moderate and severe sMR probably reflect the magnitude of the problem. Furthermore, our study did not explore the effect of drugs on the survival of patients with reduced ejection fraction, where the guideline recommends medical treatment before valve intervention.

In summary, the findings suggest that moderate or severe SMR is highly prevalent in heart failure, increases with age, and is associated with a poorer outcome. These findings indicate a considerable challenge for healthcare systems in view of the increasing elderly population. Severe sMR is associated with excessive risk of mortality overall and no heart failure subgroup is spared; sMR in patients with midrange and preserved ejection fractions, not considered in previous studies, is substantial. Despite broad population healthcare coverage and all available stateof-the-art treatment options, surgical valve repair and replacement are rarely performed owing to the associated high risk features of patients with sMR. Less invasive treatment-that is, transcatheter valve repair, which meets the clinical need for a low risk and durable treatment, is rarely used. Transcatheter mitral valve repair should be considered across the entire spectrum of heart failure to keep up with the increasing demand for treatment.

\section{Conclusions and policy implications}

With $10 \%$ overall prevalence, sMR is common among patients with heart failure, and no subgroup is spared. Surgical repair and replacement, and low risk transcatheter mitral valve repair, are rarely performed. The extent of sMR is projected to increase with an ageing population, indicating a growing demand for treatment. Echocardiographic screening and monitoring will be critical not only for diagnosis of heart failure but also for identifying high risk patients with SMR. A close collaboration between general practicians, internal medicine specialists, and specialised heart valve and heart failure teams will be key to streamlining patient flow to tackle the growing demand for treatment and provide optimal patient care.

Contributors: PEB, GG, and MHü designed the study. PEB, CG, MA, and $J M$ acquired the data. PEB, GH, MH, and GSp collected and cleaned the data. GH, GSt, MHe, PEB, and GG performed the statistical analyses. PEB wrote the first draft of the manuscript. PEB, GH, NP, MHü, GSt, GSp, SP, HA, MA, JM, CH, MHe, and GG interpreted the results and critically revised the manuscript. GG, PEB, and MHü were the study supervisors. The corresponding author attests that all listed authors meet authorship criteria and that no others meeting the criteria have been omitted. PEB, GG and MHü are the guarantors of the study.

Funding: This work was supported by a grant from the Austrian Science Fund (FWF No KLI-818B). The funders had no role in considering the study design or in the collection, analysis, interpretation of data, writing of the report, or decision to submit the article for publication.

Competing interests: All authors have completed the ICMJE uniform disclosure form at www.icmje.org/coi_disclosure.pdf and declare: this work was supported by the Austrian Science Fund (FWF). MA received proctor fees from Edwards Lifesciences and Abbott and adviser fees from Medtronic. IM received speaker fees from Edwards Lifesciences, Boston Scientific, Medtronic, and Abbott. $\mathrm{CH}$ received proctor fees, speakers bureau, and institutional grant from Edwards Lifesciences and Boston Scientific. The remaining authors have no other relationships or activities that could appear to have influenced the submitted work.

The lead author (the manuscript's guarantor) affirms that the manuscript is an honest, accurate, and transparent account of the study being reported; that no important aspects of the study have been omitted; and that any discrepancies from the study as planned (and, if relevant, registered) have been explained.

Dissemination to participants and related patient and public communities: Dissemination to patients and related public communities will be achieved directly and through official press release, social media streams, and societal meetings.

Provenance and peer review: Not commissioned; externally peer reviewed.

Ethical approval: This study was approved by the institutional review board of the Medical University of Vienna (IRBNr: 2137).

Data sharing: No additional data available.

This is an Open Access article distributed in accordance with the Creative Commons Attribution Non Commercial (CC BY-NC 4.0) license, which permits others to distribute, remix, adapt, build upon this work non-commercially, and license their derivative works on different terms, provided the original work is properly cited and the use is noncommercial. See: http://creativecommons.org/licenses/by-nc/4.0/.

1 Bartko PE, Arfsten $\mathrm{H}$, Heitzinger $\mathrm{G}$, et al. A unifying concept for the quantitative assessment of secondary mitral regurgitation. J Am Coll Cardiol 2019;73:2506-17. doi:10.1016/j.jacc.2019.02.075

2 Goliasch G, Bartko PE, Pavo N, et al. Refining the prognostic impact of functional mitral regurgitation in chronic heart failure. Eur Heart / 2018;39:39-46. doi:10.1093/eurheartj/ehx402

3 Rossi A, Dini FL, Faggiano P, et al. Independent prognostic value of functional mitral regurgitation in patients with heart failure. A quantitative analysis of 1256 patients with ischaemic and non-ischaemic dilated cardiomyopathy. Heart 2011;97:1675-80. doi:10.1136/hrt.2011.225789

4 Conrad N, Judge A, Tran J, et al. Temporal trends and patterns in heart failure incidence: a population-based study of 4 million individuals. Lancet 2018;391:572-80. doi:10.1016/S01406736(17)32520-5

5 Levy D, Kenchaiah S, Larson MG, et al. Long-term trends in the incidence of and survival with heart failure. $N$ Engl Med 2002:347:1397-402. doi:10.1056/NEJMoa020265

6 Dziadzko V, Clavel MA, Dziadzko M, et al. Outcome and undertreatment of mitral regurgitation: a community cohort study. Lancet 2018:391:960-9. doi:10.1016/S0140-6736(18)30473-2

7 d'Arcy JL, Coffey S, Loudon MA, et al. Large-scale community echocardiographic screening reveals a major burden of undiagnosed valvular heart disease in older people: the OXVALVE Population Cohort Study. Eur Heart / 2016;37:3515-22. doi:10.1093/eurheartj/ ehw229

8 Nkomo VT, Gardin JM, Skelton TN, Gottdiener JS, Scott CG, Enriquez-Sarano M. Burden of valvular heart diseases: a populationbased study. Lancet 2006;368:1005-11. doi:10.1016/S01406736(06)69208-8

9 Singh JP, Evans JC, Levy D, et al. Prevalence and clinical determinants of mitral, tricuspid, and aortic regurgitation (the Framingham Heart Study). Am J Cardiol 1999;83:897-902. doi:10.1016/S0002 9149(98)01064-9

10 Stone GW, Vahanian AS, Adams DH, et al, Mitral Valve Academic Research Consortium (MVARC). Clinical trial design principles and endpoint definitions for transcatheter mitral valve repair and replacement: Part 1: Clinical trial design principles: consensus document from the Mitral Valve Academic Research Consortium. J Am Coll Cardiol 2015;66:278-307. doi:10.1016/j. jacc.2015.05.046 
11 Stone GW, Adams DH, Abraham WT, et al, Mitral Valve Academic Research Consortium (MVARC). Clinical trial design principles and endpoint definitions for transcatheter mitral valve repair and replacement: Part 2: Endpoint definitions: a consensus document from the Mitral Valve Academic Research Consortium. J Am Coll Cardiol 2015;66:308-21. doi:10.1016/j.jacc.2015.05.049

12 Ponikowski P, Voors AA, Anker SD, et al, ESC Scientific Document Group. 2016 ESC Guidelines for the diagnosis and treatment of acute and chronic heart failure: The task force for the diagnosis and treatment of acute and chronic heart failure of the European Society of Cardiology (ESC). Developed with the special contribution of the Heart Failure Association (HFA) of the ESC. Eur Heart 12016;37:2129-200. doi:10.1093/eurhearti/ehw128

13 Rosenhek R, Binder T, Porenta G, et al. Predictors of outcome in severe, asymptomatic aortic stenosis. N Engl J Med 2000;343:6117. doi:10.1056/NEIM200008313430903

14 Lang RM, Bierig M, Devereux RB, et al, American Society of Echocardiography's Nomenclature and Standards Committee, Task Force on Chamber Quantification, American College of Cardiology Echocardiography Committee, American Heart Association, European Association of Echocardiography, European Society of Cardiology. Recommendations for chamber quantification. Eur Echocardiogr 2006;7:79-108. doi:10.1016/i.euje.2005.12.014

15 Yancy CW, Jessup M, Bozkurt B, et al, American College of Cardiology Foundation, American Heart Association Task Force on Practice Guidelines. 2013 ACCF/AHA guideline for the management of heart failure: a report of the American College of Cardiology Foundation/ American Heart Association Task Force on Practice Guidelines. / Am Coll Cardiol 2013:62:e147-239 doi:10.1016/j.jacc 2013.05.019

16 Yancy CW, Jessup M, Bozkurt B, et al. 2017 ACC/AHA/HFSA focused update of the 2013 ACCF/AHA guideline for the management of heart failure: a report of the American College of Cardiology/American
Heart Association Task Force on Clinical Practice Guidelines and the Heart Failure Society of America. J Am Coll Cardiol 2017;70:776-803. doi:10.1016/j.jacc.2017.04.025

17 Mosterd A, Hoes AW. Clinical epidemiology of heart failure. Heart 2007;93:1137-46. doi:10.1136/hrt.2003.025270

18 Bartko PE, Pavo N, Pérez-Serradilla A, et al. Evolution of secondary mitral regurgitation. Eur Heart J Cardiovasc Imaging 2018;19:622-9. doi:10.1093/ehjci/jey023

19 Mihaljevic T, Lam BK, Rajeswaran J, et al. Impact of mitral valve annuloplasty combined with revascularization in patients with functional ischemic mitral regurgitation. J Am Coll Cardiol 2007;49:2191-201. doi:10.1016/j.jacc.2007.02.043

20 Wu AH, Aaronson KD, Bolling SF, Pagani FD, Welch K, Koelling TM. Impact of mitral valve annuloplasty on mortality risk in patients with mitral regurgitation and left ventricular systolic dysfunction. J Am Coll Cardiol 2005;45:381-7. doi:10.1016/j.jacc.2004.09.073

21 Baumgartner H, Falk V, Bax JJ, et al, ESC Scientific Document Group. 2017 ESC/EACTS Guidelines for the management of valvular heart disease. Eur Heart / 2017;38:2739-91. doi:10.1093/eurheartj/ ehx391

22 Stone GW, Lindenfeld J, Abraham WT, et al, COAPT Investigators. Transcatheter mitral-valve repair in patients with heart failure. $N$ Engl I Med 2018;379:2307-18. doi:10.1056/NEJMoa1806640

23 Bartko PE, Hülsmann M, Hung J, et al. Secondary valve regurgitation in patients with heart failure with preserved ejection fraction, heart failure with mid-range ejection fraction, and heart failure with reduced ejection fraction. Eur Heart / 2020;41:2799-810. doi:10.1093/eurheartj/ehaa129

24 Obadia JF, Messika-Zeitoun D, Leurent G, et al, MITRA-FR Investigators. Percutaneous repair or medical treatment for secondary mitral regurgitation. N Engl J Med 2018;379:2297-306. doi:10.1056/NEJMoa1805374 